\title{
ASYMPTOTIC PERIODICITY OF THE ITERATES OF POSITIVITY PRESERVING OPERATORS
}

\author{
M. MIKLAVČIC
}

Abstract. Assume that

(A1) $X$ is a real Banach space.

(A2) $X^{+}$is a closed subset of $X$ with the following properties:

(i) if $x \in X^{+}, y \in X^{+}, \alpha \in[0, \infty)$ then $x+y \in X^{+}$and $\alpha x \in X^{+}$;

(ii) there exists $M_{0} \in(0, \infty)$ such that for each $x \in X$ there exist $x_{+} \in X^{+}$ and $x_{-} \in X^{+}$which satisfy

$$
x=x_{+}-x_{-}, \quad\left\|x_{+}\right\| \leq M_{0}\|x\|, \quad\left\|x_{-}\right\| \leq M_{0}\|x\|
$$

and if $x=y_{+}-y_{-}$for some $y_{+} \in X^{+}, y_{-} \in X^{+}$then $y_{+}-x_{+} \in X^{+}$;

(iii) if $x \in X^{+}, y \in X^{+}$then $\|x\| \leq\|x+y\|$.

(A3) $B$ is a bounded linear operator on $X$.

(A4) $B X^{+} \subset X^{+}$.

(A5) $F_{0}$ is a nonempty compact subset of $X$ and $\lim _{n \rightarrow \infty} \operatorname{dist}\left(B^{n} x, F_{0}\right)=0$ whenever $x \in X^{+}$and $\|x\|=1$.

Then $B^{n} x$ is asymptotically periodic for every $x \in X$. This, and other properties of $B$, are proven in the paper.

1. Introduction. It is well known that the iterates of operators with some compactness and some positivity properties are asymptotically periodic, e.g. $[\mathbf{1}, \mathbf{3}, \mathbf{4}, \mathbf{5}$, $\mathbf{7}, \mathbf{8}$. This implies that the peripheral point spectrum of the operator consists of finitely many roots of unity which is quite remarkable. Assumptions A1-A5 represent results of an attempt to isolate the crucial properties of the space and of the operator which make the asymptotic periodicity possible.

It may be somewhat surprising that the space has to satisfy only assumptions A1 and A2 [1, p. 714]; see also [7], however, this is important in the statistical theory of deterministic processes $[\mathbf{2}, \mathbf{5}]$. For example, if $\tau$ is a map of the unit interval into itself and if

$$
(B f)(x)=\frac{d}{d x} \int_{\tau^{-1}[0, x]} f(y) d y
$$

for a probability density $f \in L^{1}(0,1)$, then $B^{n} f$ describes the evolution of densities generated by the deterministic system $\left\{\tau^{n}\right\}$. Assumption A5 can be verified for some $\tau$ [5]. In certain cases, although $B^{n} f$ is not eventually periodic in $L^{1}$, it is, however, eventually periodic in a space that contains Dirac-delta functions. One would expect that such situations occur typically when the sequence $\left\{\tau^{n} x\right\}$ is eventually periodic for almost all $x$. There are plenty of such spaces which satisfy also $\mathrm{A} 1$ and $\mathrm{A} 2$. For example, we can take that $X=b a(S, \mathscr{E}, \mathbf{R})[\mathbf{1}$, p. 160], the space of bounded, real-valued, finitely additive set functions on a field $\mathscr{E}$ of subsets

Received by the editors April 10, 1986.

1980 Mathematics Subject (Classification (1985 Revision). Primary 47B55, 58F11, 47B05, 47A10. 
of a set $S$ and $X^{+}=\{\mu \in X \mid \mu(E) \geq 0$ for all $E \in \mathscr{E}\}$, or, if $\mathscr{E}$ is a $\sigma$-field, we can take $X=c a(S, \mathscr{E}, \mathbf{R})[\mathbf{1}]$, the space of countably additive members of $b a(S, \mathscr{E}, \mathbf{R})$.

Assumption A2 seems to be intuitively clear, except possibly for "and if" part in A2ii. However, if $X=\mathbf{R}^{3}, X^{+}=\left\{(x, y, z) \mid z \geq \sqrt{x^{2}+y^{2}}\right\}$ and if $B$ represents the rotation around $z$-axis by an angie equal to irrational multiple of $\pi$ then all assumptions A1-A5 are satisfied except for "and if" part in A2ii, and of course, we do not have asymptotic periodicity in this case.

Assumption A5 was introduced in [5] and a simple way to verify it is given by the following:

THEOREM 1.1. Suppose that $T$ is a bounded linear operator on a (real or complex) Banach space $W$ and that

(1) $\lim _{n \rightarrow \infty}(1 / n)\left\langle T^{n} x, y\right\rangle=0$ for all $x \in W$ and all $y \in W^{*}$,

(2) $\left\|T^{m}-K\right\|<1$ for some integer $m \geq 1$ and some compact linear operator $K$ on $W$.

Then there exist $a \in(0,1), b<\infty$, and a nonempty compact set $F \subset W$ such that $\operatorname{dist}\left(T^{n} x, F\right) \leq b a^{n}$ whenever $n \geq 1, x \in W$ and $\|x\| \leq 1$.

We write $\langle x, y\rangle$ instead of $y(x)$ whenever $x \in W$ and $y \in W^{*}$ (the dual of $W$ ). This theorem is a slightly modified version of Theorem VIII.8.3 [1] and is proven in $\S 6$. Condition 2 of the theorem was introduced in [4] and has been very often used in studies of the behaviour of the iterates of $T$, e.g. $[\mathbf{1}, \mathbf{7}, \mathbf{8}]$. Assumptions of the theorem are more restrictive than $\mathrm{A} 5$; for example, let $X=L^{p}(0,1)$ for some $1 \leq p<\infty$ and $(B f)(x)=x f(x)$ a.e. for $f \in X$.

If $(S, \mathscr{E}, \mu)$ is a positive measure space and if $W$ is any of the spaces $L^{1}(S, \mathscr{E}, \mu)$, $b a(S, \mathscr{E}, \mathbf{R}), c a(S, \mathscr{E}, \mathbf{R}), \ldots[\mathbf{1}$, p. $51 \mathbf{1}]$ then the assumption 2 of Theorem 1.1 is satisfied if $T^{n}$ is a weakly compact operator on $W$ for some $n \geq 1$. If $X=$ $L^{1}(S, \mathscr{E}, \mu)$ for some $\sigma$-finite measure space $(S, \mathscr{E}, \mu)$ and if $B$ is Markov operator on $X$ then A5 is satisfied if there is a weakly compact set $F$ in $X$ such that $\lim _{n \rightarrow \infty} \operatorname{dist}\left(B^{n} x, F\right)=0$ whenever $x \in X^{+}$and $\|x\|=1[3]$.

I wish to thank T. Y. Li for many illuminating discussions and a critical reading of the manuscript.

2. Results. Assumptions A1 through A5 will be in effect throughout the rest of the paper. Define

$$
M=\sup _{n \geq 0}\left\|B^{n}\right\|
$$

$$
Y=\left\{y \in X \mid y=\lim _{i \rightarrow \infty} B^{n_{i}} x \text { for some } x \in X \text { and some } 1<n_{1}<n_{2}<\cdots\right\} \text {. }
$$

THEOREM 2.1. $M<\infty$ and $Y$ is finite-dimensional vector space. If $Y=\{0\}$ then $\lim _{n \rightarrow \infty}\left\|B^{n} x\right\|=0$ for every $x \in X$.

This theorem and some other important properties of $Y$ are proved in $\S 3$. Define $N=\operatorname{dim} Y \geq 0$ and let $E$ be the set of all $x \in X^{+} \cap Y$ such that $\|x\|=1$ and if $x=y+z$ for some $y \in X^{+} \cap Y, z \in X^{+} \cap Y$ then $y=t x$ for some $t \in[0,1]$. Some results of $\S 4$ are represented in the following theorem. 
THEOREM 2.2. $E$ is a set of $N$ elements and if $N \geq 1$ then $E$ is a basis for $Y$. Moreover

(1) If $x \in E$ then $\|B x\| \geq M^{-1}$ and $B x=\|B x\| y$ for some $y \in E$.

(2) If $\|B\|=1$ then $\|B x\|=1$ for all $x \in E$.

(3) If $x \in E, y \in E, x \neq y$ and $h \in X^{+}$are such that $x-h \in X^{+}$and $y-h \in X^{+}$ then $\lim _{n \rightarrow \infty}\left\|B^{n} h\right\|=0$, moreover, if $\|B\|=1$ then $\|x-h\|=\|y-h\|=1$.

Note that part 3 is commenting on "disjoint support" of elements of $E$.

If $N \geq 1$ let $e_{1}, \ldots, e_{N}$ be an enumeration of $E$. In this case define $p:\{1, \ldots, N\}$ $\rightarrow\{1, \ldots, N\}$ and $\lambda:\{1, \ldots, N\} \rightarrow(0,\|B\|]$ by

$$
B e_{i}=\lambda(i) e_{p(i)} \text {. }
$$

$p$ is one-to-one (Lemma 4.6). Let $m_{0}$ be the smallest positive integer such that $m_{0}$ th iterate of $p$ is the identity map. We have that $m_{0} \leq e^{N / e}$ and that $B^{m_{0}} x=x$ for all $x \in E$ (Lemma 4.7). Observe that if $x=e_{1}+B e_{1}+\cdots+B^{m_{0}-1} e_{1}$ then $x \in X^{+},\|x\| \geq 1$ by $\mathrm{A} 2$ iii and $B x=x$.

THEOREM 2.3. Suppose that $N \geq 1$. Then there exist $f_{1}, \ldots, f_{N}$ in $X^{*}$ such that for all $i, j \in\{1, \ldots, N\}$

(1) $\lim _{n \rightarrow \infty}\left\|B^{n}\left(x-\sum_{k=1}^{N}\left\langle x, f_{k}\right\rangle e_{k}\right)\right\|=0$ for all $x \in X$,

(2) $\left\langle e_{i}, f_{j}\right\rangle=\delta_{i j}$ (Kronecker delta),

(3) $0 \leq\left\langle x, f_{i}\right\rangle \leq M\|x\|$ for all $x \in X^{+}$,

(4) $\left\|f_{i}\right\| \leq M M_{0}$

(5) $B^{*} f_{p(i)}=\lambda(i) f_{i}$,

(6) $\lim _{n \rightarrow \infty}\left\langle x, B^{* n}\left(y-\sum_{k=1}^{N}\left\langle e_{k}, y\right\rangle f_{k}\right)\right\rangle=0$ for all $x \in X$ and all $y \in X^{*}$.

This is our main theorem. It is proven in $\S 5$. Observe that if $M=M_{0}=1$ then $f_{i}$ is actually a positive tangent functional to $e_{i}$ and hence, in some spaces $X, f_{i}$ is uniquely determined by $e_{i}$. The following theorem concerning the spectrum of $B$ is also proven in $\S 5$ and it implies that if $N \geq 1$ then

$$
Y=\left\{x \in X \mid B^{m_{0}} x=x\right\}=\bigcup_{n=1}^{\infty}\left\{x \in X \mid B^{n} x=x\right\} .
$$

THEOREM 2.4. Suppose that

$$
B^{k} x=\cos \varphi x-\sin \varphi y, \quad B^{k} y=\sin \varphi x+\cos \varphi y
$$

for some integer $k \geq 1, \varphi \in[0,2 \pi)$ and some $x \in X, y \in X$ such that $\|x\|+\|y\|>0$. Then $N \geq 1, x \in Y, y \in Y$ and $\varphi=2 \pi n / m_{0}$ for some $n \in\left\{0,1, \ldots, m_{0}-1\right\}$.

The following theorem has applications in the study of the Boltzmann equation $[\mathbf{5}, \mathbf{6}]$ and is proven in $\S 5$.

THEOREM 2.5. For each $x \in X$ there exist $x_{1} \in Y$ and a unique $x_{0} \in Y$ such that

$$
\lim _{n \rightarrow \infty}\left\|B^{n}\left(x-x_{0}\right)\right\|=\lim _{n \rightarrow \infty}\left\|\frac{1}{n} \sum_{i=0}^{n-1} B^{i} x-x_{1}\right\|=0
$$


moreover,

$$
\lim _{t \rightarrow \infty}\left\|e^{-t} e^{B t} x-x_{1}\right\|=0,
$$

$B x_{1}=x_{1}$ and if $N \geq 1$ then $x_{1}=m_{0}^{-1} \sum_{i=0}^{m_{0}-1} B^{i} x_{0}$.

For $x \in X$ define $P_{0} x=x_{0}, P_{1} x=x_{1}$ where $x_{0}$ and $x_{1}$ correspond to $x$ as in the Theorem 2.5. $P_{0}$ and $P_{1}$ are projections and both commute with $B$. The following theorem is proven in $\S 6$.

THEOREM 2.6. If $\left\|B^{m}-K\right\|<1$ for some integer $m \geq 1$ and some compact linear operator $K$ then there exist $a \in(0, \infty)$ and $b \in(0, \infty)$ such that

$$
\left\|B^{n}\left(I-P_{0}\right)\right\| \leq b e^{-a n}, \quad\left\|\frac{1}{n} \sum_{i=0}^{n-1} B^{i}-P_{1}\right\| \leq \frac{b}{n}, \quad\left\|e^{-t} e^{B t}-P_{1}\right\| \leq b e^{-a t}
$$

for all $n \geq 1$ and all $t>0$.

3. Properties of $Y$. Since $F_{0}$ is a bounded set, A5 and A2ii imply that $\left\{\left\|B^{n} x\right\| n \geq 0\right\}$ is bounded for every $x \in X$. The principle of uniform boundedness implies that $M<\infty$.

For $x \in X$ define

$$
Q(x)=\left\{y \mid y=\lim _{i \rightarrow \infty} B^{n_{i}} x \text { for some } 1<n_{1}<n_{2}<\cdots\right\} .
$$

Note that $Y=\bigcup_{x \in X} Q(x)$ and if $y \in Q(x)$ then $B y \in Q(x)$ and $\|y\| \leq M\|x\|$; hence $B Y \subset Y$.

LEMMA 3.1. If $x \in X$ then every sequence in $\left\{B^{n} x \mid n \geq 0\right\}$ has a subsequence that converges to some element in $X$.

Proof. If $x \in X^{+}$and $\|x\|=1$ then $\left\|B^{n} x-x_{n}\right\|<\operatorname{dist}\left(B^{n} x, F_{0}\right)+1 / n$ for some $x_{n} \in F_{0}$ and all $n \geq 1$. A2ii and the fact that $F_{0}$ is compact imply the lemma.

LEMMA 3.2. If $x_{0} \in X, x \in Q\left(x_{0}\right), y \in Q\left(x_{0}\right)$ then $y \in Q(x)$ and $\|y\| \leq M\|x\|$.

PROOF. Pick $\varepsilon>0, m \geq 1$ and note that $\left\|x-B^{n_{1}} x_{0}\right\|<\varepsilon /(1+M)$, $\| y-$ $B^{n_{2}} x_{0} \|<\varepsilon /(1+M)$ for some $n_{1}>1$ and some $n_{2}>n_{1}+m$. Therefore $\| y-$ $B^{n_{2}-n_{1}} x \|<\varepsilon$.

LEMMA 3.3. If $x_{0} \in X$ and $x_{1}, x_{2}, \ldots$ are in $Q\left(x_{0}\right)$ then there exist $1<n_{1}<$ $n_{2}<\cdots$ and $x \in Q\left(x_{0}\right)$ such that $\lim _{i \rightarrow \infty} x_{n_{i}}=x$.

PROOF. Pick $1<m_{1}<m_{2}<\cdots$ such that $\left\|B^{m_{i}} x_{0}-x_{i}\right\|<1 / i$ for $i \geq 1$ and apply Lemma 3.1 .

Above lemmas imply the following.

LEMMA 3.4. If $x \in X$ then

(1) $Q(x)$ is a compact set,

(2) $Q(x)=\{0\}$ iff inf $\{\|y\| y \in Q(x)\}=0$ iff $\lim _{n \rightarrow \infty} B^{n} x=0$,

(3) if $y \in Q(x)$ then $Q(y)=Q(x)$,

(4) $x \in Y$ iff $x \in Q(x)$,

(5) if $x \in X^{+}$then $Q(x) \subset X^{+}$.

LEMMA 3.5. If $x \in Y, n \geq 0$, then $x=B^{n} y$ for some $y \in Q(x)$.

PROOF. By Lemma $3.4 x=\lim _{i \rightarrow \infty} B^{n_{i}} x$ for some $n<n_{1}<n_{2}<\cdots$. Apply Lemma 3.1 to $B^{n_{i}-n} x$. 
LEMMA 3.6. If $x \in Y, y \in Y, \alpha \in \mathbf{R}, \beta \in \mathbf{R}$ then $\alpha x+\beta y \in Y$.

PROOF. By Lemma 3.5 there exist $x_{n} \in Q(x), y_{n} \in Q(y)$ such that $x=B^{n} x_{n}$, $y=B^{n} y_{n}$ for $n \geq 1$. Lemma 3.3 implies that $\lim _{i \rightarrow \infty} x_{n_{i}}=x_{0}, \lim _{i \rightarrow \infty} y_{n_{i}}=y_{0}$ for some $x_{0} \in Q(x), y_{0} \in Q(y), n_{1}<n_{2}<\cdots ;$ hence

$$
\alpha x+\beta y=\lim _{i \rightarrow \infty} B^{n_{i}}\left(\alpha x_{0}+\beta y_{0}\right) .
$$

LEMMA 3.7. If $x \in Y, y \in Y, n \geq 0$ and $B^{n} x=B^{n} y$ then $x=y$.

ProOF. Lemmas 3.6, 3.4 imply that $x-y \in Q(x-y)=\{0\}$.

LEMMA 3.8. For each $x \in X$ there exists a unique $x_{0} \in Y$ such that

$$
\lim _{n \rightarrow \infty} B^{n}\left(x-x_{0}\right)=0
$$

moreover, $x_{0} \in Q(x)$.

ProOF. Pick $n_{1}<n_{2}<\cdots$ and $y \in Q(x)$ such that $\lim _{i \rightarrow \infty} B^{n_{i}} x=y$. Let $y_{n} \in Q(x)$ be such that $y=B^{n} y_{n}$ for $n \geq 1$ (Lemmas 3.5, 3.4). Lemma 3.3 implies that (by renaming a subsequence) we may assume that $\lim _{i \rightarrow \infty} y_{n_{i}}=x_{0}$ for some $x_{0} \in Q(x)$. If $m>n_{i}$ then

$$
\left\|B^{m}\left(x-x_{0}\right)\right\| \leq M\left\|B^{n_{i}} x-y\right\|+M\left\|y_{n_{i}}-x_{0}\right\|
$$

and therefore $\lim _{n \rightarrow \infty} B^{n}\left(x-x_{0}\right)=0$. If $\lim _{n \rightarrow \infty} B^{n}(x-z)=0$ for some $z \in Y$ then $B^{n}\left(z-x_{0}\right)=B^{n}\left(x-x_{0}\right)-B^{n}(x-z) \rightarrow 0$ as $n \rightarrow \infty$, so, $Q\left(z-x_{0}\right)=\{0\}$ by Lemma 3.4 and since $z-x_{0} \in Q\left(z-x_{0}\right)$ we have $z=x_{0}$.

LEMMA 3.9. If $x \in Y, y \in Q(x)$ and $y-x \in X^{+}$then $y=x$.

PROOF. Define $y_{1}=y, y_{0}=x, z_{0}=y-x \in X^{+} \cap Y$ and suppose that $z_{0} \neq 0$. Since $z_{0} \in Q\left(z_{0}\right)$ we have by Lemma 3.4 that $\sigma=\inf \left\{\|z\| \mid z \in Q\left(z_{0}\right)\right\}>0$ and that $Q\left(z_{0}\right) \subset X^{+}$. Suppose that we have found $y_{0}, y_{1}, \ldots, y_{k+1}$ in $Q(x)$ and $z_{0}, z_{1}, \ldots, z_{k}$ in $Q\left(z_{0}\right)$ for some $k \geq 0$ such that $y_{i+1}=y_{i}+z_{i}$ for $0 \leq i \leq k$. By Lemma 3.2 $y_{k+1}=\lim _{i \rightarrow \infty} B^{n_{i}} y_{k}$ for some $n_{1}<n_{2}<\cdots$ and by choosing a subsequence we may assume (Lemma 3.1) that both $B^{n_{i}} y_{k+1}$ and $B^{n_{i}} z_{k}$ converge. Define $y_{k+2}=\lim _{i \rightarrow \infty} B^{n_{i}} y_{k+1}, z_{k+1}=\lim _{i \rightarrow \infty} B^{n_{i}} z_{k}$. By Lemma $3.4 y_{k+2} \in Q(x)$ and $z_{k+1} \in Q\left(z_{0}\right)$ and also $y_{k+2}=y_{k+1}+z_{k+1}$. Therefore there exist $y_{0}, y_{1}, y_{2}, \ldots$ in $Q(x)$ and $z_{0}, z_{1}, z_{2}, \ldots$ in $Q\left(z_{0}\right)$ such that $y_{k+1}=y_{0}+z_{0}+\cdots+z_{k}$ for all $k \geq 0$. Assumption A2iii implies that if $0 \leq n<m$ then $\left\|y_{m}-y_{n}\right\|=\left\|z_{n}+\cdots+z_{m-1}\right\| \geq$ $\left\|z_{n}\right\| \geq \sigma>0$ and this contradicts Lemma 3.3 ; therefore $z_{0}=0$.

LEMMA 3.10. If $x \in X, y \in Q(x)$ and $y-x \in X^{+}$then $\lim _{n \rightarrow \infty} B^{n}(y-x)=0$.

PROOF. Let $z=y-x$ and pick $n_{1}<n_{2}<\cdots, z_{1} \in Q(z), y_{1} \in Q(y), x_{1} \in Q(x)$ such that $B^{n_{i}} z \rightarrow z_{1}, B^{n_{i}} y \rightarrow y_{1}, B^{n_{i}} x \rightarrow x_{1}$ as $i \rightarrow \infty$. So, $x_{1} \in Y, y_{1} \in Q\left(x_{1}\right)$ by Lemma 3.4, $z_{1}=y_{1}-x_{1} \in Q(z) \subset X^{+}$and Lemma 3.9 implies that $z_{1}=0$. Lemma 3.4 implies that $B^{n} z \rightarrow 0$ as $n \rightarrow \infty$. that

THEOREM 3.11. For each $x \in Y$ there exist $z_{+} \in X^{+} \cap Y, z_{-} \in X^{+} \cap Y$ such

$$
x=z_{+}-z_{-}, \quad\left\|z_{+}\right\| \leq M M_{0}\|x\|, \quad\left\|z_{-}\right\| \leq M M_{0}\|x\|
$$

and if $x=y_{+}-y_{-}$for some $y_{+} \in X^{+} \cap Y, y_{-} \in X^{+} \cap Y$ then $y_{+}-z_{+} \in X^{+}$. 
ProOF. Take $x_{+}, x_{-}$as in A2ii. $x=x_{+}-x_{-}=\lim _{i \rightarrow \infty} B^{n_{i}}\left(x_{+}-x_{-}\right)$for some $n_{1}<n_{2}<\cdots$. We may assume (by renaming a subsequence of $n_{i}$ ) that $\lim _{i \rightarrow \infty} B^{n_{i}} x_{ \pm}=z_{ \pm}$for some $z_{ \pm} \in Q\left(x_{ \pm}\right)$. By Lemma 3.10

$$
\lim _{n \rightarrow \infty} B^{n}\left(z_{+}-x_{+}\right)=0 .
$$

If $x=y_{+}-y_{-}$for some $y_{ \pm} \in X^{+} \cap Y$ then $g:=y_{+}-x_{+} \in X^{+}$, so, $y_{+}-$ $z_{+}=g+x_{+}-z_{+}$and since $y_{+}-z_{+} \in Q\left(y_{+}-z_{+}\right)$there are $m_{1}<m_{2}<\cdots$ such that $y_{+}-z_{+}=\lim _{i \rightarrow \infty} B^{m_{i}}\left(y_{+}-z_{+}\right)=\lim _{i \rightarrow \infty}\left(B^{m_{i}} g-B^{m_{i}}\left(z_{+}-x_{+}\right)\right)=$ $\lim _{i \rightarrow \infty} B^{m_{i}} g \in X^{+}$.

ProOF OF THEOREM 2.1. All that we still have to show is that $\operatorname{dim} Y<\infty$. Let $F_{1}$ denote the closed convex hull of $F_{0} \cup\{0\}$. Note that $F_{1}$ is compact, convex and $0 \in F_{1}$. Define

$$
S^{+}=\left\{x \in X^{+} \cap Y \mid\|x\| \leq 1\right\} .
$$

If $x \in S^{+} \backslash\{0\}$ let $y=x /\|x\|$, and since $y \in Y$ there are $n_{1}<n_{2}<\cdots$ such that $y=\lim _{i \rightarrow \infty} B^{n_{i}} y$; hence $y \in F_{0}$ by A5 and $x=\|x\| y+(1-\|x\|) 0 \in F_{1}$. Therefore $S^{+} \subset F_{1}$ and $\bar{S}^{+}$is compact. Define

$$
\begin{gathered}
S_{1}=\left\{x \in X \mid x=y-z \text { for some } y \in \bar{S}^{+}, z \in \bar{S}^{+}\right\}, \\
S=\left\{x \in \bar{Y} \mid M M_{0}\|x\| \leq 1\right\} .
\end{gathered}
$$

Clearly, $\bar{S}_{1}$ is totally bounded and hence it is compact. Theorem 3.11 implies that $S \subset \bar{S}_{1}$ and hence $S$ is compact. Thus $S$ is compact in $\bar{Y}$ and therefore $\operatorname{dim} Y<\infty$.

4. Properties of $E$. If $N=0$ then $E=\varnothing$, so, assume $N \geq 1$ throughout this section.

LEMMA 4.1. $E$ is not empty and $\left\{\sum_{i=1}^{N+1} \alpha_{i} x_{i} \mid \alpha_{i} \in[0, \infty), x_{i} \in E\right.$ for $i=$ $1, \ldots, N+1\}$ is dense in $X^{+} \cap Y$.

ProOF. Let $x_{1}, \ldots, x_{N}$ be a basis of $Y$. Define $T: \mathbf{R}^{N} \rightarrow Y$ by

$$
T\left(\alpha_{1}, \ldots, \alpha_{N}\right)=\sum_{i=1}^{N} \alpha_{i} x_{i} .
$$

Define $C=T^{-1} X^{+}$. The following properties of $C$ will be needed.

(1) If $x \in C, y \in C, \alpha \in[0, \infty)$ then $x+y \in C, \alpha x \in C$.

(2) If $x \in C$ and $-x \in C$ then $x=0$ (by A2iii).

(3) $C$ is closed and $C \neq\{0\}$ (by Theorem 3.11 and $N>0$ ).

Let $C_{1}$ be the convex hull of $\{x \in C \mid\|x\|=1\}$. Note that $C_{1}$ is nonempty, compact and the property 2 of $C$ implies that $0 \notin C_{1}$. Hahn-Banach theorem gives us $y_{0} \in \mathbf{R}^{N}$ and $\gamma \in \mathbf{R}$ such that

$$
0<\gamma<\left(x, y_{0}\right)
$$

for all $x \in C_{1}$. Hence $\left(x, y_{0}\right) \geq \gamma\|x\|$ for all $x \in C$. Define $D=\left\{x \in C \mid\left(x, y_{0}\right)=1\right\}$. $D$ is nonempty, compact and convex. Let $E_{1}$ be the set of extreme points of $D$. By verification

$$
E=\left\{\frac{1}{\|T x\|} T x \mid x \in E_{1}\right\} .
$$


The Krein-Milman theorem implies that

$$
\left\{\sum_{i=1}^{N+1} \alpha_{i} x_{i} \mid \alpha_{i} \geq 0, x_{i} \in E_{1} \text { for } i=1, \ldots, N+1 \text { and } \sum_{i=1}^{N+1} \alpha_{i}=1\right\}
$$

is dense in $D$ and this completes the proof.

LEMMA 4.2. Suppose that $x_{1}, \ldots, x_{k}$ are distinct elements of $E$. If $\left(\alpha_{1}, \ldots, \alpha_{k}\right)$ $\in \mathbf{R}^{k}$ and $\sum_{i=1}^{k} \alpha_{i} x_{i} \in X^{+}$then $\alpha_{i} \geq 0$ for $i=1, \ldots, k$.

Proof. Let $P_{k}$ be the lemma as stated. $P_{1}$ is implied by A2iii. Assume that $k>1$ and $P_{k-1}$ is true. Suppose $\left(\alpha_{1}, \ldots, \alpha_{k}\right) \in \mathbf{R}^{k}, y=\sum_{i=1}^{k} \alpha_{i} x_{i} \in X^{+}$and $\alpha_{j}<0$ for some $j$. Since $\sum_{i \neq j} \alpha_{i} x_{i}=y-\alpha_{j} x_{j} \in X^{+}, P_{k-1}$ implies $\alpha_{i} \geq 0$ whenever $i \neq j$; and A2iii implies $\alpha_{m}>0$ for some $m$. Define $x=\alpha_{m} x_{m}-y$ and let $z_{+}, z_{-}$be as in Theorem 3.11 ; hence

$$
x=\alpha_{m} x_{m}-y=-\sum_{i \neq m} \alpha_{i} x_{i}=z_{+}-z_{-} .
$$

$P_{k-1}$ and $j \neq m$ imply $z_{+} \neq 0$. Theorem 3.11 implies $\alpha_{m} x_{m}-z_{+} \in X^{+} \cap Y$ and $-\alpha_{j} x_{j}-z_{+} \in X^{+} \cap Y$. Definition of $E$ implies $z_{+}=t x_{m}$ and $z_{+}=r x_{j}$ for some $t \in\left(0, \alpha_{m}\right], r \in\left(0,-\alpha_{j}\right]$ and therefore $x_{m}=x_{j}$. Contradiction.

LEMMA 4.3. E contains precisely $N$ elements and these form a basis for $Y$.

ProOF. Let $x_{1}, \ldots, x_{k}$ be distinct elements of $E$. Lemma 4.2 and A2iii imply that if $x=\sum_{i=1}^{k} \alpha_{i} x_{i} \in X^{+}$then $0 \leq \alpha_{i} \leq\|x\|$ for $i=1, \ldots, k$. Thus $x_{1}, \ldots, x_{k}$ are linearly independent; hence $|E| \leq N$. Assume $k=|E|$. By Lemma 4.1 $S=$ $\left\{\sum_{i=1}^{k} \beta_{i} x_{i} \mid \beta_{i} \in[0, \infty)\right.$ for $\left.i=1, \ldots, k\right\}$ is dense in $X^{+} \cap Y$, and because $\sum_{i=1}^{k} \beta_{i}^{2} \leq$ $k\left\|\sum_{i=1}^{k} \beta_{i} x_{i}\right\|^{2}$ whenever $\beta_{i} \in[0, \infty)$ for $i=1, \ldots, k$ we have that $S=X^{+} \cap Y$. Theorem 3.11 implies $\operatorname{span}\left\{x_{1}, \ldots, x_{k}\right\}=Y$ and $k=N$.

LEMMA 4.4. If $x \in E$ then $\|B x\|>0$ and $B x=\|B x\| y$ for some $y \in E$.

ProOF. Lemma 3.7 implies $\|B x\|>0$. Let $y=\|B x\|^{-1} B x$ and suppose $y=$ $u+v$ for some $u, v$ in $X^{+} \cap Y$. Lemma 3.5 implies $u=B u_{1}$ and $v=B v_{1}$ for some $u_{1} \in Q(u) \subset X^{+}, v_{1} \in Q(v) \subset X^{+}$. Lemma 3.7 implies $x=\|B x\| u_{1}+\|B x\| v_{1}$; hence $\|B x\| u_{1}=t x$ for some $t \in[0,1]$, so, $u=t y$ and $y \in E$.

LEMMA 4.5. If $x \in E, n \geq 1, \lambda \in \mathbf{R}$ and $B^{n} x=\lambda x$ then $\lambda=1$.

Proof. $|\lambda| \leq 1$ because $M<\infty$. Lemma 3.4 implies $x \in Q(x) \neq\{0\}$ and therefore $|\lambda|=1$. A2iii implies $\lambda=1$.

LEMMA 4.6. $p$ is one-to-one.

PROOF. If $p(i)=p(j)$ then $B \lambda(i)^{-1} e_{i}=B \lambda(j)^{-1} e_{j}$ and by Lemma $3.7 \lambda(i)^{-1} e_{i}$ $=\lambda(j)^{-1} e_{j}$; hence $i=j$.

LEMMA 4.7. $m_{0} \leq e^{N / e}$ and $B^{m_{0}} x=x$ for every $x \in E$. Moreover, $M^{-1} \leq$ $\|B x\| \leq\|B\|$ for every $x \in E$.

PROOF. Since $p$ is one-to-one $m_{0}$ is well defined and $m_{0} \leq e^{N / e}$. Lemma 4.5 implies $B^{m_{0}} x=x$ for all $x \in E$. If $x \in E$ then $1=\left\|B^{m_{0}-1} B x\right\| \leq M\|B x\|$. 
LEMMA 4.8. If $x \in E, y \in E, x \neq y$ and $h \in X^{+}$are such that $x-h \in X^{+}$and $y-h \in X^{+}$then $\lim _{n \rightarrow \infty} B^{n} h=0 ;$ moreover, if $\|B\|=1$ then $\|x-h\|=\|y-h\|=1$.

PROOF. Let $f=x-h, g=y-h$ and note that $x=B^{n m_{0}} f+B^{n m_{0}} h$ and $y=B^{n m_{0}} g+B^{n m_{0}} h$ for $n \geq 0$. Lemma 3.1 implies that there are $f_{0}, g_{0}, h_{0}$ in $X^{+} \cap Y$ and $n_{1}<n_{2}<\cdots$ such that $B^{n_{i} m_{0}} f \rightarrow f_{0}, B^{n_{i} m_{0}} g \rightarrow g_{0}, B^{n_{i} m_{0}} h \rightarrow h_{0}$ as $i \rightarrow \infty$. Therefore $x=h_{0}+f_{0}$ and $y=h_{0}+g_{0}$. Definition of $E$ implies $h_{0}=0$ and by Lemma $3.4 \lim _{n \rightarrow \infty} B^{n} h=0$. Since $x \in Q(f)$ we have $1 \leq M\|f\|=M\|x-h\| \leq M$ and similarly $1 \leq M\|y-h\| \leq M$.

\section{Asymptotic periodicity.}

PROOF OF THEOREM 2.3. For $x \in X$ and $1 \leq i \leq N$ define $\gamma_{i}(x)$ by $\sum_{i=1}^{N} \gamma_{i}(x) e_{i}=x_{0}$ where $x_{0}$ is as in Lemma 3.8. Lemmas 3.8, 4.2 imply that $\gamma_{i}(x) \geq 0$ if $x \in X^{+}$and $1 \leq i \leq N$. If $x \in X, y \in X, \alpha \in \mathbf{R}, \beta \in \mathbf{R}$ then

$$
\begin{aligned}
& \left\|B^{n}\left(\alpha x+\beta y-\sum_{i=1}^{N}\left(\alpha \gamma_{i}(x)+\beta \gamma_{i}(y)\right) e_{i}\right)\right\| \\
& \quad \leq|\alpha|\left\|B^{n}\left(x-\sum_{i=1}^{N} \gamma_{i}(x) e_{i}\right)\right\|+|\beta|\left\|B^{n}\left(y-\sum_{i=1}^{N} \gamma_{i}(y) e_{i}\right)\right\|
\end{aligned}
$$

and therefore $\gamma_{i}(\cdot)$ are linear functionals. If $x \in X$ then (Lemma 3.4) for some $n_{1}<n_{2}<\cdots$

$$
\left\|\sum_{i=1}^{N} \gamma_{i}(x) e_{i}\right\|=\lim _{j \rightarrow \infty}\left\|B^{n_{j}} \sum_{i=1}^{N} \gamma_{i}(x) e_{i}\right\|=\lim _{j \rightarrow \infty}\left\|B^{n_{j}} x\right\| \leq M\|x\| .
$$

Thus, A2iii implies that $0 \leq \gamma_{j}(x) \leq\left\|\sum_{i=1}^{N} \gamma_{i}(x) e_{i}\right\| \leq M\|x\|$ for $x \in X^{+}, 1 \leq j \leq$ $N$. A2ii implies that $\left|\gamma_{j}(x)\right| \leq M M_{0}\|x\|$ for all $1 \leq j \leq N, x \in X$. Define $f_{i} \in X^{*}$ by $\left\langle x, f_{i}\right\rangle=\gamma_{i}(x)$ for $x \in X, 1 \leq i \leq N$. This proves parts $1,2,3,4$ of the theorem. Since

$$
\begin{aligned}
B x-\sum_{i=1}^{N}\left\langle B x, f_{i}\right\rangle e_{i} & =B x-\sum_{i=1}^{N}\left\langle x, \lambda(i)^{-1} B^{*} f_{p(i)}\right\rangle \lambda(i) e_{p(i)} \\
& =B\left(x-\sum_{i=1}^{N}\left\langle x, \lambda(i)^{-1} B^{*} f_{p(i)}\right\rangle e_{i}\right)
\end{aligned}
$$

for every $x \in X$ part 5 is proven. Part 5 implies

$$
\sum_{k=1}^{N}\left\langle x, f_{k}\right\rangle\left\langle B^{n} e_{k}, y\right\rangle=\sum_{k=1}^{N}\left\langle x, B^{* n} f_{k}\right\rangle\left\langle e_{k}, y\right\rangle
$$

and

$$
\left\langle x, B^{* n}\left(y-\sum_{k=1}^{N}\left\langle e_{k}, y\right\rangle f_{k}\right)\right\rangle=\left\langle B^{n}\left(x-\sum_{k=1}^{N}\left\langle x, f_{k}\right\rangle e_{k}\right), y\right\rangle
$$

whenever $x \in X, y \in X^{*}$ and $n \geq 1$. This proves part 6 . 
PROOF OF THEOREM 2.4. Note that for $n \geq 1$

$$
\begin{gathered}
B^{n k} x=\cos (n \varphi) x-\sin (n \varphi) y, \\
B^{n k} y=\sin (n \varphi) x+\cos (n \varphi) y, \\
\inf _{\beta \geq 0}\{\|\cos \beta x-\sin \beta y\|+\|\sin \beta x+\cos \beta y\|\}>0 .
\end{gathered}
$$

Theorem 2.1 implies $Y \neq\{0\}$ and hence $N \geq 1$. Pick $u \in Y, v \in Y$ such that $\lim _{n \rightarrow \infty} B^{n}(x-u)=\lim _{n \rightarrow \infty} B^{n}(y-v)=0$. Note that $B^{m_{0}} u=u, B^{m_{0}} v=v$. Define $\alpha=m_{0} \varphi$ and

$$
\begin{array}{ll}
u_{j}:=\cos (j \alpha) x-\sin (j \alpha) y=B^{j k m_{0}} x \rightarrow u & \text { as } j \rightarrow \infty, \\
v_{j}:=\sin (j \alpha) x+\cos (j \alpha) y=B^{j k m_{0}} y \rightarrow v & \text { as } j \rightarrow \infty .
\end{array}
$$

Therefore $u_{j \pm 1}=\cos \alpha u_{j} \mp \sin \alpha v_{j}$ and $v_{j \pm 1}=\cos \alpha v_{j} \pm \sin \alpha u_{j}$ for $j \geq 2$ and $\|u\|+\|v\|>0$. This implies that $\cos \alpha=1, \sin \alpha=0, x=u, y=v$.

LEMMA 5.1. If $m \geq 1,0 \leq n \leq m-1, t>0$ then

$$
\left|e^{-t} \sum_{k=0}^{\infty} \frac{t^{k m+n}}{(k m+n) !}-\frac{1}{m}\right| \leq \frac{m-1}{m} \exp \left(-t\left(1-\cos \frac{2 \pi}{m}\right)\right) .
$$

PROOF. If $z=\exp (2 \pi i / m)$ then

$$
m e^{-t} \sum_{k=0}^{\infty} \frac{t^{k m+n}}{(k m+n) !}=\sum_{k=0}^{m-1} z^{-k n} \exp \left(z^{k} t-t\right) .
$$

ProOF OF THEOREM 2.5. Existence and uniqueness of $x_{0}$ is given in Lemma 3.8. Note that for $n \geq 1, t>0$

$$
\begin{gathered}
\frac{1}{n} \sum_{k=0}^{n-1} B^{k} x=\frac{1}{n} \sum_{k=0}^{n-1} B^{k}\left(x-x_{0}\right)+\frac{1}{n} \sum_{k=0}^{n-1} B^{k} x_{0} \\
e^{-t} e^{B t} x=e^{-t} \sum_{k=0}^{\infty} \frac{t^{k}}{k !} B^{k}\left(x-x_{0}\right)+e^{-t} \sum_{k=0}^{\infty} \frac{t^{k}}{k !} B^{k} x_{0}
\end{gathered}
$$

and that both first sums converge to 0 as $n \rightarrow \infty, t \rightarrow \infty$. Thus, if $Y=\{0\}$ take $x_{1}=0$ and if $Y \neq\{0\}$ then let $x_{1}=m_{0}^{-1} \sum_{k=0}^{m_{0}-1} B^{k} x_{0}$ and observe that for $n \geq 1$

$$
\left\|\frac{1}{n} \sum_{k=0}^{n-1} B^{k} x_{0}-x_{1}\right\| \leq \frac{2 m_{0} M\left\|x_{0}\right\|}{n}
$$

and that Lemma 5.1 implies for $t>0$

$$
\left\|e^{-t} \sum_{k=0}^{\infty} \frac{t^{k}}{k !} B^{k} x_{0}-x_{1}\right\| \leq\left(m_{0}-1\right) M\left\|x_{0}\right\| \exp \left(-t\left(1-\cos \frac{2 \pi}{m_{0}}\right)\right) .
$$

This completes the proof.

6. Quasi-compactness. If $W$ is a Banach space let $\mathscr{L}(W)$ denote the set of all bounded linear maps from $W$ into $W$. If $W$ is complex Banach space and $C: W \rightarrow W$ is such that $C^{2}=I,\|C x\|=\|x\|$ and $C(\alpha x+\beta y)=\bar{\alpha} x+\bar{\beta} y$ for all $x \in W, y \in W, \alpha \in \mathbf{C}, \beta \in \mathbf{C}$ then $C$ is called conjugation on $W$. The following theorem follows directly from the Theorem VIII.8.3 [1], see also [8]. 
THEOREM 6.1. Suppose that $W$ is a complex Banach space, $T \in \mathscr{L}(W)$, $\lim _{n \rightarrow \infty}\left\langle T^{n} x, y\right\rangle / n=0$ for all $x \in W$ and all $y \in W^{*}$, and $\left\|T^{m}-K\right\|<1$ for some integer $m \geq 1$ and some compact $K \in \mathscr{L}(W)$. Then there exist $K_{1} \in \mathscr{L}(W)$, $V_{1} \in \mathscr{L}(W), a \in(0,1), b<\infty$ such that

(1) $\operatorname{Ran}\left(K_{1}\right)$ is finite dimensional and $\sup _{n \geq 0}\left\|K_{1}^{n}\right\|<\infty$,

(2) $\left\|V_{1}^{n}\right\| \leq b a^{n}$ for $n=1,2, \ldots$,

(3) $T^{n}=K_{1}^{n}+V_{1}^{n}$ for $n=1,2, \ldots$,

(4) if $C$ is a conjugation on $W$ and $T C=C T$ then $K_{1} C=C K_{1}$,

(5) if $x_{0} \in \operatorname{Ran}\left(K_{1}\right)$ then there exist $x_{1}, \ldots, x_{n}$ in $\operatorname{Ran}\left(K_{1}\right)$ and $\lambda_{1}, \ldots, \lambda_{n}$ in $\mathbf{C}$ such that $x_{0}=x_{1}+\cdots+x_{n}, K_{1} x_{i}=T x_{i}=\lambda_{i} x_{i},\left|\lambda_{i}\right|=1$ for $1 \leq i \leq n$.

PROOF OF THEOREM 1.1. If $W$ is complex then the statement is obvious. Assume $W$ is real. Let $Z=W \times W, Z_{1}=W^{*} \times W^{*}$ be the usual complexifications of $W, W^{*}$ defined by

$$
\|(x, y)\|=\sup \left\{\sqrt{\|\alpha x-\beta y\|^{2}+\|\beta x+\alpha y\|^{2}} \mid \alpha \in \mathbf{R}, \beta \in \mathbf{R}, \alpha^{2}+\beta^{2}=1\right\} .
$$

Define $V=T^{m}-K, T_{0}(x, y)=(T x, T y), K_{0}(x, y)=(K x, K y), V_{0}(x, y)=$ $(V x, V y), C_{1}(x, y)=(y, x), C_{2}(x, y)=(x,-y)$ for $(x, y) \in Z$. It is easy to verify that $T_{0}, K_{0}, V_{0}$ are in $\mathscr{L}(Z), K_{0}$ is compact, $V_{0}=T_{0}^{m}-K_{0},\left\|V_{0}\right\|=\|V\|<1$ and $C_{1}$ and $C_{2}$ are conjugations on $Z$ and both commute with $T_{0}$. If $x \in Z, y \in Z^{*}$ then

$$
\left\langle T_{0}^{n} x, y\right\rangle=\left\langle T^{n} x_{r}, y_{r}\right\rangle-\left\langle T^{n} x_{i}, y_{i}\right\rangle+i\left\langle T^{n} x_{r}, y_{i}\right\rangle+i\left\langle T^{n} x_{i}, y_{r}\right\rangle
$$

for some $\left(x_{r}, x_{i}\right) \in Z,\left(y_{r}, y_{i}\right) \in Z_{1}$. Let $K_{1} \in \mathscr{L}(Z), V_{1} \in \mathscr{L}(Z), a \in(0,1)$, $b<\infty$ be as given by Theorem 6.1. Applying $C_{1}$ and $C_{2}$ one can show that $K_{1}(x, y)=\left(K_{2} x, K_{2} y\right), V_{1}(x, y)=\left(V_{2} x, V_{2} y\right)$ for some $V_{2} \in \mathscr{L}(W)$, some compact $K_{2} \in \mathscr{L}(W)$ and all $(x, y) \in Z$; moreover, $\left\|V_{2}^{n}\right\|=\left\|V_{1}^{n}\right\| \leq b a^{n},\left\|K_{2}^{n}\right\|=\left\|K_{1}^{n}\right\|$, $T^{n}=K_{2}^{n}+V_{2}^{n}$ for $n \geq 1$. Let $F$ be the closure of $K_{2}\left\{x \in W \mid\|x\| \leq \sup _{n \geq 0}\left\|K_{2}^{n}\right\|\right\}$.

PROOF OF THEOREM 2.6. Let $Z=X \times X$ and $Y_{c}=Y \times Y$ be the complexifications of $X$ and $Y$ (as above). Define $B_{0}(x, y)=(B x, B y), K_{0}(x, y)=(K x, K y)$ for $(x, y) \in Z$. Let $K_{1} \in \mathscr{L}(Z), V_{1} \in \mathscr{L}(Z), a \in(0,1), b<\infty$ be as in Theorem 6.1 (corresponding to $\left.B_{0}\right)$. As above $K_{1}(x, y)=\left(K_{2} x, K_{2} y\right), V_{1}(x, y)=\left(V_{2} x, V_{2} y\right)$ for some $K_{2} \in \mathscr{L}(X), V_{2} \in \mathscr{L}(X)$ and all $(x, y) \in Z$.

Suppose $x_{0} \in \operatorname{Ran}\left(K_{1}\right)$ and let $x_{1}, \ldots, x_{n}, \lambda_{1}, \ldots, \lambda_{n}$ be as in Theorem 6.1. Since $\left|\lambda_{i}\right|=1$ and $B_{0} x_{i}=\lambda_{i} x_{i}$ for $1 \leq i \leq n$ Theorem 2.4 implies that $x_{0} \in Y_{c}$ and, clearly, $B_{0} x_{0}=K_{1} x_{0}$. This implies that if $x \in \operatorname{Ran}\left(K_{2}\right)$ then $x \in Y$ and $K_{2} x=B x$.

Pick $x \in X, n \geq 1$. Then $B^{n} x=K_{2}^{n} x+V_{2}^{n} x=B^{n-1} K_{2} x+V_{2}^{n} x$ and since $K_{2} x \in Y$ there exists (Lemma 3.5) $x_{0} \in Y$ such that $K_{2} x=B x_{0}$. Therefore $B^{n} x=B^{n} x_{0}+V_{2}^{n} x$ and by Lemma 3.8

$$
\left\|B^{n}\left(I-P_{0}\right)\right\|=\left\|V_{2}^{n}\right\| \leq b a^{n} .
$$

If this inequality is used in the proof of Theorem $2.5(\S 5)$ then the other two inequalities are obtained.

\section{REFERENCES}

1. N. Dunford and J. T. Schwartz, Linear operators, Part 1, 4th printing, Interscience, New York, 1967.

2. F. Hofbauer and G. Keller, Ergodic properties of invariant measures for piecewise monotonic transformations, Math. Z. 180 (1982), 119140. 
3. J. Komornik, Asymptotic periodicity of the iterates of weakly constrictive Markov operators, Tôhoku Math. J. 38 (1986), 15-27.

4. N. Kryloff and N. Bogoliouboff, Sur les propriétés en chaine, C. R. Acad. Sci. Paris 204 (1937), 1386-1388.

5. A. Lasota, T. Y. Li and J. A. Yorke, Asymptotic periodicity of the iterates of Markov operators, Trans. Amer. Math. Soc. 286 (1984), 751-764.

6. M. Miklavðið, On limit states of a linearized Boltzmann equation, SIAM J. Math. Anal. 19 (1988), 150-152.

7. H. H. Schaefer, On positive contractions in $L^{p}$ spaces, Trans. Amer. Math. Soc. 257 (1980), 261-268.

8. K. Yoshida and S. Kakutani, Operator-theoretical treatment of Markoff process and mean ergodic theorem, Ann. of Math. (2) 42 (1941), 188-228.

Department of Mathematics, Michigan State University, East Lansing, MICHIGAN 48824 